\title{
Sars-Cov-2 Rapid Antibody Tests: Interest and Limits
}

\section{Naamoune S*, Salah S, Ait Ali Yahia CT and Radi H \\ Department of Immunology, Mustapha university hospital center, Algeria}

*Corresponding author: Soumia Naamoune, Mustapha university Hospital, Algiers, Algeria, Tel: +213550959271; +213658432724; Email: s.naamoune@gmail.com

$1^{\text {st }}$ author (Naamoune soumia) and the $2^{\text {nd }}$ (Salah Sofiane Samir) contributed equally to this work

\section{Abstract}

Since the onset of COVID 19 outbreak last December 2019, several groups of scientists have tried to characterize the novel coronavirus's structure, to develop fast immunoassays, easier to use in order to control the current situation. Nowadays, hundreds of serological immunoassays are commercialized; therefore, we aim to compare four POCT according to their particularities, sensitivities and specificities too. However, some limits have been observed. Consequently, it is highly important to establish strict recommendations for their indication of use.

Keywords: Rapid Tests; SARS-CoV2; Serological Tests; COVID 19; POCT

\section{Introduction}

Severe acute respiratory syndrome coronavirus-2 (SARS-CoV-2) has been widely spread since its emergence in December 2019 in Wuhan, China [1-4]. The diagnosis of Corona Virus Disease 19(COVID-19) is mainly based on the clinical manifestations, epidemiological history and also, some auxiliary examinations such as RT-qPCR and CT scanner [1]. In response to this global epidemic, many immunoassays have been offered by manufacturers such as ELISA and rapid tests (POCT: point-of care immunodiagnostic test) $[1,2]$. The purpose of these tests is to detect the antigen in the respiratory samples or in the blood of human antibodies (IgG or IgM) produced against SARS-Cov-2 virus [5].

Rapid serological assays can be used easily outside laboratories, their execution and interpretation do not require qualified staff and a result can be obtained in a very short time. Currently, hundreds of tests are available [6]. However, what are the particularities of these tests and what place do they really occupy in the management of this new pandemic?.

\section{Characteristics of Rapid Antibody Tests}

The main peculiarity of a rapid serological test is the speed of its execution and the ease of its interpretation, which could allow rapid mass screening to initiate an effective health control policy [7]. So, the manufacture of commercial rapid test kits was immediately started as soon as the molecular structure of SARS-Cov-2 was characterized. The principal of most of those commercial kits is based on the colloidal gold immunochromatography assay in order to detect antibodies directed against SARS-CoV2 $[8,9]$. But, during covid 19, the antibodies detected by the different methods can be directed against the Nucleocapsid (N), the whole Spike (S) antigen, the S2 subunit of the Spike protein which is common to all coronaviruses or the S1 subunit which is specific to each strain of coronavirus [10].

Several type of kits are available and depending on the isotype of the antibody detected, the companies offer a screening for combined IgM/IgG, or a detection of IgM and IgG separately, while some others offer a screening for IgM alone. The essay is realized according to the producers' recommendations, either on whole blood which allows their use even at home, or on serum or plasma. However, it is imperative to respect proper specimen handling and storage to maintain the performance of serologic tests.

\section{Example of Some Rapid Antibody Tests}

In this short review, we took the example of 4 kits produced by four different companies; the principle of the 4 
kits is based on the colloidal gold immunochromatography technology with different variations of the technique. In the Well's reaction, the test sample interacts with the "Detection buffer" containing different components from a producer to another one, nitrocellulose strips are also coated differently from a producer to another one too. For the first kit, antichicken IgY polyclonal antibody and recombinant SARSCov-2 antigen, marked the both of them with colloid gold are coated in reagent conjugate pad. Anti-human IgG monoclonal antibody and anti-human IgM monoclonal antibody are coated in test zone of nitrocellulose membrane to bind with the immune complex (specific SARS-Cov-2 antibody contained in the sample/colloid gold marked recombinant SARS-Cov-2 antigen) and form a line in test area (Test line). Chicken IgY is coated in the control area of the strip and bind with the excess of colloid gold marked anti-chicken IgY polyclonal antibody (Control line). For the second one, it uses double antigen sandwich method. The detection line was coated with SARS-Cov- 2 recombinant antigen, and the control line was coated with goat polyclonal IgG. During the assay, the antibody anti SARS-Cov- 2 in the sample binds first to the colloid gold labeled Cov recombinant antigen to form a solid phase Cov antigen-Cov antibody-labeled Cov recombinant antigen-colloidal gold complex, at the control line position form a solid phase goat polyclonal IgG-labelled Cov recombinant antigen-colloidal gold complex; after completing the test, we observe the Colloidal gold color reaction of Test line and Control line which determine the result. For the third kit, detection of IgM and IgG is separated. During the test, the sample and the detection buffer are applied in the Well reaction, the IgG or IgM anti SARS-Cov-2 here in the sample combined with Colloidal gold-labelled SARS-Cov-2 recombinant antigen forming a complex. During the flow, the complexes move among the strip. When passing the detection line of IgM or IgG anti sars-Cov-2, the complexes are captured by anti-human IgM (Test line IgM) or anti-human IgG (Test line IgG) resulting colouring on lines; when passed the control line, colloidal gold-labelled DNP is captured by quality-control antibody resulting colouring on the line too. Regarding the last one, only screening for IgM is possible. In fact, when the sample contains the SARS-Cov-2 IgM antibody, it forms a complex with colloidal gold-labelled SARS-Cov-2 recombinant antigen. After the migration, the complexes combine with the coated Mouse anti-human IgM monoclonal antibodies at the test area and develop a colour. Goat anti-rabbit antibodies are coated in the control line to capture the gold labelled quality control antibody (Rabbit IgG antibody) and form a complex viewable with a gold coloration.

Apart the minor differences in the composition of kits and the principle of the assays mentioned above, some other elements vary from a manufacturer to another one, such as: the biological nature of the sample to be tested, the sensitivity and specificity (Table 1). Concerning the first point and according to the recommendations of each producer, the tests can be done on plasma, serum or whole blood, however, the second company does not recommend the use of whole blood, resulting on limit uses of their kits outside laboratories.

\begin{tabular}{|c|c|c|c|c|}
\hline & Sinocare ${ }^{\circledR}$ & Hotgen $®$ & Biotime $®$ & Hightop $®$ \\
\hline Detected antibody & $\operatorname{IgM} / \operatorname{IgG}$ & IgM/ IgG & IgM and IgG separatly & IgM \\
\hline \multirow{3}{*}{ Nature of the sample } & -Serum & -Serum & -Serum & -Serum \\
\hline & - Plasma & -Plasma & -Plasma & -Plasma \\
\hline & - Whole blood & & - Whole blood & - Whole blood \\
\hline \multirow{12}{*}{ Sensitivity } & \multirow{8}{*}{ Whole blood 95\% } & \multirow{12}{*}{ Not mentionned } & Whole blood & \multirow{12}{*}{$86,76 \%$} \\
\hline & & & $\mathrm{gM}: 64,3 \%$ & \\
\hline & & & IgG: $96,4 \%$ & \\
\hline & & & IgM+IgG: $96,4 \%$ & \\
\hline & & & Serum & \\
\hline & & & IgM: $81,7 \%$ & \\
\hline & & & IgG: $95,8 \%$ & \\
\hline & & & IgM+IgG: $96,3 \%$ & \\
\hline & \multirow{4}{*}{ Serum/Plasma 96.3\% } & & Plasma & \\
\hline & & & IgM:67,9\% & \\
\hline & & & IgG: $96,4 \%$ & \\
\hline & & & IgM+IgG: $96,4 \%$ & \\
\hline
\end{tabular}




\begin{tabular}{|c|c|c|c|c|}
\hline \multirow{11}{*}{ Specifity } & \multirow{3}{*}{ Whole blood $99.2 \%$} & \multirow{11}{*}{ Not mentionned } & Whole blood IgM: $100 \%$ & \multirow{11}{*}{$96,15 \%$} \\
\hline & & & IgG: 98,7\% & \\
\hline & & & IgM+IgG: 98,7\% & \\
\hline & \multirow{8}{*}{$\begin{array}{c}\text { Serum/Plasma } \\
99.6 \%\end{array}$} & & Serum & \\
\hline & & & IgM: $98,3 \%$ & \\
\hline & & & IgG: $95 \%$ & \\
\hline & & & IgM+IgG: 95\% & \\
\hline & & & Plasma & \\
\hline & & & IgM: $96,7 \%$ & \\
\hline & & & IgG: $93,5 \%$ & \\
\hline & & & IgM+IgG: 93,5\% & \\
\hline
\end{tabular}

Table 1: Characteristics of 04 rapid antibody tests'kits.

\section{Limitations of Rapid Antibody Tests}

Although, the rapid surgical assays have multiple advantages, the WHO does not recommend the use of "POCT" in the diagnosis of covid 19 and considers that a larger number of studies on more significant cohorts is necessary to pose this indication [5]. These apprehensions can be explained by the limits of these tests. Indeed, the positivity of the serological tests depends on the seroconversion because the IgM can be detected at the earliest, 3 days after the start of the infection and reach their maximum 2 to 3 weeks later, while the detection of the IgG remains variable; it can be synchronous with that of IgM or occurs later with a peak around the $17^{\text {th }}$ day from the infection's starting date of [1113]. Note that, the time of onset of seroconversion pends on several factors: age, sex, nutritional status, disease's severity, medication taking and immune status [5]. Furthermore, a lower level of antibodies not reaching the detection threshold of the used test is another point to be taken into consideration. As a result, a negative test does not completely exclude the infection by SARS-COV-2 [5]. In the other hand, we could wrongly conclude that a positive test indicates the patient was previously infected with SARS-CoV-2 because of possible cross reactions. In order to overcome this bias, some laboratories use recombinant SARS-CoV-2 S1 subunit antigen which shows few cross-reaction with others Coronavirus [14]. Regarding the 04 kits cited in this review, no details were provided by the manufacturers about the nature of the antigen used for their assays and finally, rapid serological tests are qualitative and cannot quantify the level of antibodies.

In addition, at this moment, it is well established the evaluation of the sensitivity and specificity of the different serogicals assays must be similar and meet standardized and rigorous recommendations. This is unfortunately not the case for the rapid serological tests used for the detection of anti SARS-Cov-2 Antibodies available nowadays, on the market.

\section{Conclusion}

To manage the actual COVID 19 pandemic, the use of rapid serological assays cannot be yet considered as a tool for diagnosis and cannot at any case replace RT-qPCR. Furthermore, any inappropriate use can have disastrous consequences for public health. Therefore it is important to emphasize the limit of these tests and the need to interpret them cautiously. Their use at home by general public must be prohibited. However, WHO encourages experts to use them in the context of epidemiological research and clinical surveillance.

\section{References}

1. Li X, Geng M, Peng Y, Meng L, Lu S (2020) Molecular immune pathogenesis and diagnosis of COVID-19. J Pharm Anal 10(2): 102-108.

2. Nuccetelli M, Pieri M, Grelli S, Ciotti M, Miano R, et al. (2020) SARS-CoV-2 infection serology: a useful tool to overcome lockdown?. Cell Death Discovry 6(1): 38.

3. Chen N, Zhou M, Dong X, Qu J, Gong F, et al. (2020) Epidemiological and clinical characteristics of 99 cases of 2019 novel coronavirus pneumonia in Wuhan, China: a descriptive study. The Lancet 395(10223): 507-513.

4. Amanat F, Stadlbauer $\mathrm{D}$, Strohmeier $\mathrm{S}$, Nguyen $\mathrm{T}$, Chromikova V, et al. (2020) A serological assay to detect SARS-CoV-2 Seroconversion in humans. Nature Medicine.

5. (2020) Advice on the use of point-of-care immunodiagnostic tests for COVID-19: Scientific Brief. 
World Health Organization.

6. Sheridan C (2020) Fast, portable tests come online to curb coronavirus pandemic. Nat Biotechnol 38(5): 515518.

7. Winter AK, Hegde S (2020) The important role of serology for COVID-19 control. Lancet Infect Dis.

8. Prod'hom G, Bille J (2008) Infectious Disease Diagnosis: place of point of care tests (POCT). Rev Med Suisse 4: 908-913.

9. Pin Lee CY, Lin RTP, Renia L, Ng LFP (2020) Serological Approaches for COVID-19: Epidemiologic Perspective on Surveillance and Control. Frontiers Immunology 11: 879.
10. Torres R, Rinder HM (2020) Double-Edged SpikeAre SARS-CoV-2 Serologic Tests Safe Right Now?. Lab Medicine 51(3): 236-238.

11. Jalali Nadoushan $M$, Ahmadi S, Jalali Nadoushan $P$ (2020) Serology Testing for SARS-CoV-2: Benefits and Challenges. Iranian Journal of Pathology 15(3): 154-155.

12. Cui J, Li F, Shi ZL (2019) Origin and evolution of pathogenic coronaviruses. Nat Rev Microbiol 17(3): 181-192.

13. Zhao J, Yuan Q Wang H, Liu W, Liaoet X, et al. (2020) Antibody responses to SARS-CoV-2 in patients of novel coronavirus disease 2019. Clin Infect Dis.

14. Lv H, Wu NC, Tsang OTY, Yuan M, Perera RAPM, et al. (2020) Crossreactive antibody response between SARSCoV-2 and SARS-CoV infections. BioRxiv. 